\title{
NUMERICAL STUDY OF AN UNSTEADY NON-PREMIXED FLAME IN A POROUS MEDIUM BASED ON THE THERMAL EQUILIBRIUM MODEL
}

\author{
Watit Pakdee, Thammasathit Sakkarangkoon \\ Center for RED on Energy Efficiency in Thermo-Fluid Systems (CETF), Faculty of Engineering, Thammasat University, \\ Klong Nueng, Klong Lueng, Pathumtani, Thailand \\ e-mail: pwatit@engr.tu.ac.th
}

\begin{abstract}
Non-premixed combustion in porous media, also known as diffusion combustion, is found in many burners and combustion systems for both household and industry. However, in terms of porous combustion, there have been far fewer studies of non-premixed as compared to premixed combustion. Moreover, most of the previous works regarding the non-premixed porous combustion were based on experiments. The present research numerically investigates the behavior of non-premixed combustion of $\mathrm{CH}_{4}$ in a porous medium. The mathematical model proposed consists of conservation of mass, momentum equation, energy equation and species equations. This set of equations is discretized based on a sixth-order accurate compact finite difference algorithm. The discretized equations are integrated according to the third-order Runge-Kutta method. The porous medium is defined as a pseudohomogeneous medium in which temperature gradients between two phases are assumed negligible. The proposed unsteady model is successfully validated with the published study. The model is able to correctly describe physical behavior of a non-premixed flame. The effects of porous materials on the combustion process are analyzed. It is found that porous combustion has a broader reaction zone than combustion in a free space. The location of the reaction zone is determined by diffusion velocity of the mixture gas. The porous structure made of $\mathrm{SiC}$ gives higher temperature than when $\mathrm{Al}_{2} \mathrm{O}_{3}$ is used since $\mathrm{SiC}$ has higher thermal conductivity and lower heat capacity. The developed model can be utilized as a tool for fast adaption of combustion systems and for optimizing the combustion efficiency.
\end{abstract}

Keywords: porous combustion, non-premixed combustion, local thermal equilibrium, pseudohomogeneous, numerical model

\section{Notations}

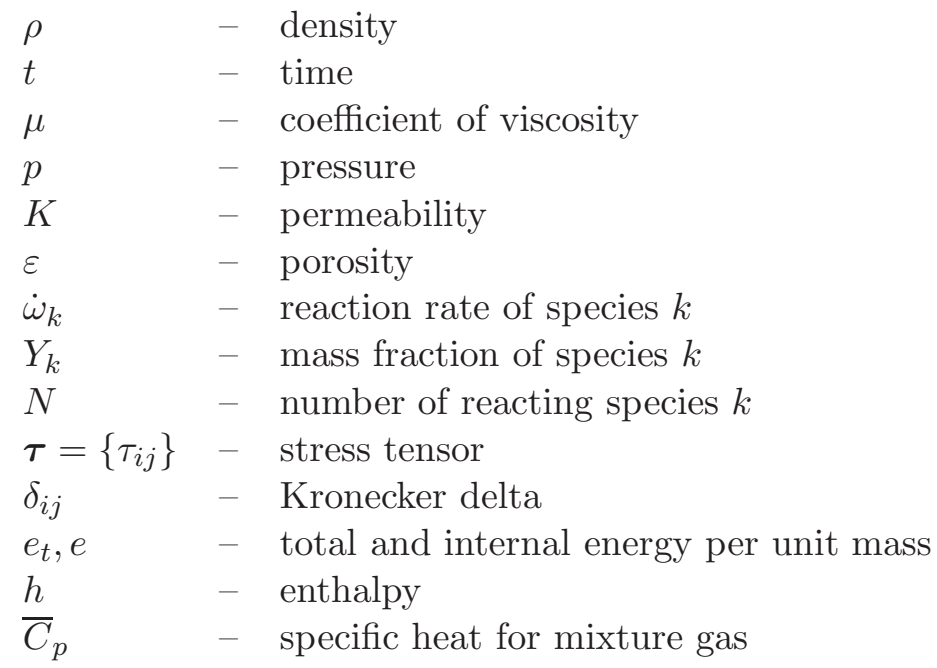




$\begin{array}{ll}T & - \text { temperature } \\ q_{j} & - \text { heat fluxes } \\ \lambda_{\text {eff }}, \lambda_{s} & - \text { effective and solid thermal conductivity } \\ \mathrm{Pr} & - \text { Prandtl number } \\ V_{k j} & - \text { Fickian velocity } \\ S_{c} & - \text { Schmidt number } \\ D_{k} & - \text { diffusion coefficient for species } k \\ n & - \text { number of iterations } \\ f & - \text { temperature dependent function } \\ R & - \text { mixture gas constant }\end{array}$

\section{Introduction}

Porous combustion has been extensively used in many current industrial applications due to many advantages over conventional or free space combustion. Combustion in porous media gives efficient energy recirculation, better flame stabilization with leaner flame stability limit, as well as a higher combustion rate. Additionally, reduction of $\mathrm{CO}$ and $\mathrm{NO}_{x}$ can be achieved. Due to many advantageous features of porous combustion, a large number of numerical simulations have been carried out to study combustion in porous media for various different aspects such as properties of porous media, porous geometry, flame stabilization, formation of pollutants, flame structure, flame speed, conversion efficiency of heat into radiation energy, etc. Simulations developed from mathematical models enable a numerical parametric study for applications that porous combustion is involved. A two-dimensional model of two different geometries of the porous burner was developed to study the effect of multidimensionality on flames within the pore scale (Hackert et al., 1999). The conservation equations solved include both gas-phase and solid-phase energy equations. A one-step global reaction mechanism for the complete combustion of the fuel was utilized. The density was obtained from the ideal gas law. The conservation equations were solved using the alternating direction implicit (ADI) method, and the pressure field was solved using the SIMPLE algorithm. Brenner et al. (2000) computed heat flow in porous media based on the pseudohomogeneous heat transfer and the flow model which treated the solid and fluid phases as an artificial unique phase. The two-dimensional steady problem of a chemically reacting mixture gas including 20 species was considered. The thermodynamic data was obtained from the Chemkin-II database (Kee et al., 1992). The finite volume code by Brenner et al. (2000) was used again to find the optimal amount of the inert components $\mathrm{H}_{2} \mathrm{O}$ and $\mathrm{HCl}$ synthesis based on the heat balance of the reactor for the $\mathrm{H}_{2} / \mathrm{Cl}_{2}$ system (Wawrzinek et al., 2001). It was found further that the flame speed and the adiabatic flame temperature were higher for the $\mathrm{H}_{2} / \mathrm{Cl}_{2}$ reaction compared to $\mathrm{CH}_{4}$ /air combustion. Effects of porous properties have also been investigated extensively. Bubnovich et al. (2007) carried out one-dimensional simulations on combustion behavior within the two layers of different sizes of alumina balls. Zeldovich's mechanism was utilized for modeling the formation of $\mathrm{NO}_{x}$. Species fractions, gas and solid temperatures were solved using Newton's method while pressure was computed by the congradient method.

A porous burner with an integrated heat exchanger was modeled as a two-dimensional axially symmetric geometry (Malico et al., 2000). The combustion reaction was described by the skeletal mechanism. The non-thermal equilibrium was considered between the gas phase and the solid phase. The chemical reaction rates and thermophysical properties were obtained using Chemkin-II. The SIMPLE method was employed to obtain numerical solutions. Further, the mathematical model accounting for turbulence effects was proposed to study one-dimensional turbulent combustion of methane/air in a porous medium. The thermo-mechanical models based 
on the double-decomposition concept were developed and used to solve the similar problem. In this work, the turbulence was predicted utilizing the macroscopic $k-\varepsilon$ model.

Nonetheless, the previous work on the modeling of the non-premixed or diffusion combustion in a porous burner is rather limited. In 2007, the numerical database of turbulent nonpremixed flame of wood pyrolysis gas was developed using direct numerical simulation (DNS) (Pakdee and Mahalingam, 2007). Combustion of a pyrolysis gas with the air was modeled by a reduced kinetic mechanism. Later, numerical simulation of methane/air non-premixed combustion in porous media was investigated (Tarokh et al., 2009). The results showed that the combustion efficiency was improved in porous media with significantly lower $\mathrm{NO}_{x}$ and $\mathrm{CO}$ emissions. Additionally, effects of swirl on combustion and radiation flux from a nonpremixed flame porous burner were investigated (Kamal and Mohamad, 2005). It was found that the gap distance between the swirling flow and the base of the porous medium could be adjusted so that the optimized radiation flux was achieved.

Recently, non-premixed combustion of porous biomass particles in a counter-flow configuration has been modeled using a derived analytical model. In this study, the combustion process was divided into different zones including pre-heat, reaction and post-flame zones (Hosseinzadeh et al., 2020). The investigations of the flame structure and extinction limits of confined diffusion flames were conducted (Endo Kokubun et al., 2017). Effects of the porosity and mass injection rate on flame extinction were determined based on the general theory by Cheatham and Matalon (2000). The chemistry considered was a global one-step reaction with a generic representation of the fuel and oxidizer. Klayborworn and Pakdee (2019) numerically investigated the effects of porous insertion in a burner on flame characteristics of turbulent non-premixed syngas combustion. It was found that the porosity and porous thickness significantly influence the flame characteristics and heat process.

From the literature, as compared with premixed combustion, the previous studies on non-premixed combustion are few. Therefore, in the present work, non-premixed combustion where the flame is stabilized and burned completely inside the porous matrix is investigated using the proposed mathematical model. To the best knowledge of the authors, the proposed model with the current approach has not been reported in the literature.

\section{Mathematical formulation}

The numerical model includes fully compressible, chemically reacting continuity, energy, Navier-Stokes and species transport equations. The transient model treats the methane combustion kinetics using a one-step reaction including four species which are $\mathrm{CH}_{4}, \mathrm{O}_{2}, \mathrm{CO}_{2}$ and $\mathrm{H}_{2} \mathrm{O}$.

\subsection{Governing equations}

In the present study, the porous medium is assumed to be homogeneous and thermally isotropic. Such a model can be used if the mass flow density, pore diameter and porosity are not too high and if the heat transport properties and the temperature are not too low. This model was successfully employed in the investigation of methane-air non-premixed combustion in porous media (Brenner et al., 2000). Numerical calculations were consistent with experimental data. Further, the same model was used to numerically investigate porous combustion for HCI synthesis (Wawrzinek et al., 2001) wherein the adiabatic flame temperature and flame speed were successfully carried out. Accordingly, the saturated fluid within the medium was considered in a local thermodynamic equilibrium (LTE) with the solid matrix (Pakdee and Rattanadecho, 2011). The validity regime of local thermal equilibrium assumption was established (Marafie and Vafai, 2001). The fluid flow was unsteady, laminar and incompressible. The pressure work and viscous dissipation were all assumed negligible. The thermophysical properties of the porous 
medium were taken to be constant. The Darcy-Forchheimer-Brinkman model was used to represent the fluid transport within the porous medium (Marafie and Vafai, 2001). Brinkmann's and Forchheimer's extensions treated the viscous stresses at the bounding walls and the non-linear drag effect due to the solid matrix respectively (Marafie and Vafai, 2001). Furthermore, the solid matrix was made of spherical particles, while the porosity and permeability of the medium were assumed to be uniform throughout the rectangular domain.

We neglect body forces, the Soret and Dufour effects and gas radiation. Although a one dimensional problem is considered in this work, the transport equations expressed below are in generalized forms for multi-dimensional systems. Equations (2.1) are conservation of mass, momentum, energy and species transport equations, respectively

$$
\begin{aligned}
& \frac{\partial \rho}{\partial t}+\frac{\partial\left(\rho u_{j}\right)}{\partial x_{j}}=0 \\
& \frac{\partial \rho u_{i}}{\partial t}+\frac{\partial \rho u_{i} u_{j}}{\partial x_{j}}=-\frac{\partial p}{\partial x_{i}}+\frac{\partial \tau_{i j}}{\partial x_{j}}-\frac{\mu}{K} u_{j}-\frac{\rho}{K} u_{j}^{2} \\
& \frac{\partial \rho e_{t}}{\partial t}+\frac{\partial\left[\left(\rho e_{t}+p\right) u_{j}\right]}{\partial x_{j}}=\frac{\partial\left(u_{j} \tau_{i j}\right)}{\partial x_{i}}-\frac{\partial q_{j}}{\partial x_{j}}+\varepsilon \sum_{k=1}^{N_{s}} \dot{\omega}_{k} \Delta h_{f, k}^{0} \\
& \frac{\partial \rho Y_{k}}{\partial t}+\frac{\partial\left(\rho Y_{k} u_{j}\right)}{\partial x_{j}}=-\frac{\partial\left(\rho Y_{k} V_{k j}\right)}{\partial x_{j}}+\varepsilon \dot{\omega}_{k} \quad k=1,2, \ldots, N
\end{aligned}
$$

where $Y_{k}$ is the mass fraction of species $k$ in the gaseous mixture. $N$ is the number of reacting species $k$ excluding $N_{2}$. Therefore, $N=4$. The mass fraction of $N_{2}$ can be found by using the constraint that summation of the mass fractions of all the species is one (Pakdee and Mahalingam, 2003). The symbols $K$ and $\varepsilon$ denote permeability and porosity of the medium, respectively.

The stress tensor is given by

$$
\tau_{i j}=\mu\left(\frac{\partial u_{i}}{\partial x_{j}}+\frac{\partial u_{j}}{\partial x_{i}}-\frac{2}{3} \delta_{i j} \frac{\partial u_{k}}{\partial x_{k}}\right)
$$

The total energy per unit mass is

$$
e_{t}=e+\frac{1}{2} \sum_{k=1}^{1} u_{k}^{2}
$$

where $e$ is the internal energy per unit mass

$$
e=\sum_{k=1}^{4} Y_{k} h_{k}-\frac{p}{\rho}
$$

The enthalpy $h$ is computed taking into account the fluid and solid portions of the porous medium as

$$
\begin{aligned}
& h_{k}=h_{k}^{0}+\varepsilon \int_{T_{0}}^{T} C_{p k}\left(T^{\prime}\right) d T^{\prime}+(1-\varepsilon) C_{p s} T \\
& C_{p_{\text {eff }}}=\varepsilon \bar{C}_{p}+(1-\varepsilon) C_{p s}
\end{aligned}
$$

The specific heat for the mixture gas is given by

$$
\bar{C}_{p}(T)=\sum_{k=1}^{4} Y_{k} C_{p k}(T)
$$


Heat fluxes $q_{j}$ amount to

$$
q_{j}=-\lambda_{\text {eff }} \frac{\partial T}{\partial x_{j}}+\rho \sum_{k=1}^{4} h_{k} Y_{k} V_{k j}
$$

where the effective thermal conductivity is

$$
\lambda_{e f f}=\frac{\varepsilon \mu \bar{C}_{p}}{\operatorname{Pr}}+(1-\varepsilon) \lambda_{s}
$$

And the Fickian velocity based on Fick's law of diffusion is expressed by

$$
V_{k j}=-\varepsilon D_{k} \frac{1}{Y_{k}} \frac{\partial Y_{k}}{\partial x_{j}}
$$

The diffusive flux of each species is a function of its concentration gradient. The diffusion model with the Schmidt approximation number $S_{c}$ by Smooke and Giovangigli (1991) is used to model the diffusion coefficient for individual species $k$ as

$$
D_{k}=\frac{\mu}{\rho S_{c k}}
$$

\subsection{Numerical approach}

In the present study, we consider nonpremixed flames of the methane-air mixture. A one dimensional $6.0 \mathrm{~cm}$ domain with a uniform finite difference grid resolution of 256 found to be adequate to resolve the smallest reaction zones, is utilized. Gaseous methane from the left side reacts with oxygen gas from the right side in the domain. The iterative finite difference method is used to solve the transient governing equations subject to their corresponding initial and boundary conditions. Approximation of the convective terms is based on an upwind finite differencing scheme, which correctly represents the directional influence of the disturbance. Spatial derivatives are discretized using a sixth-order accurate compact finite-different scheme (Lele, 1992). Such a scheme accurately models the behavior of the acoustic wave and ensures a reasonable spectral resolution for both the amplitude and phase of the solution. Simulations at the boundary and in the nodes adjacent to the boundary have the third and fourth order of accuracy, respectively. A third-order Runge-Kutta scheme is applied to integrate the set of equations in time. Although the problem presently studied is one-dimensional, this approach can be used for multi-dimensional systems. For a two-dimensional system, all the governing equations can be written in the form

$$
\frac{\partial f(x, y, t)}{\partial t}=Q(f, x, y, t)
$$

where $f$ denotes any of the dependent variables of the flow system and the solution to this variable at time $t=t 1$ is represented by $f(x, y, t 1)$. The term $Q(f, x, y, t)$ is the non-linear operator of $f$ that does not depend explicitly on $t$, and is the summation of values of all other terms in the particular governing equation. The value of the function $Q$ at time $t=t 1$ depends only on the solution function $f(x, y, t 1), x, y$ and $t$. That is, once the solution function $f$ is determined at a certain time, the function $Q$ can then be evaluated using the finite difference equations for the first and second derivatives. The solution $f$ at the next time step can be computed in three steps through the Runge-Kutta integration procedure.

The Courant-Friedrichs-Lewy (CFL) criterion is used for variable time-stepping. For a given CFL number, the time step is determined according to the following criterion

$$
\Delta t=\min (\Delta t f, \Delta t c)
$$


where $\Delta t f$ and $\Delta t c$ are the fluid and chemical times. The CFL numbers for the fluid and chemical scales are chosen to be 0.6 and 0.005, respectively (Pakdee and Mahalingam, 2003).

Since the internal energy $e$ is a function of temperature via equation (2.4) and the temperature is not known either, the internal energy cannot be computed explicitly. In the present study, the internal energy and temperature can be obtained simultaneously using the Newton-Raphson method, an iterative root finding method. We set up the function $f$ from the definition of internal energy as

$$
f=e-\sum_{k=1}^{4} Y_{k} \int_{T_{0}}^{T} C_{p k}\left(T^{\prime}\right) d T^{\prime}+R T
$$

The derivative of $f$ with respect to $T$ is

$$
f^{\prime}=-\bar{C}_{p}(T)+R
$$

where $\bar{C}_{p}(T)$ is the specific heat of the gaseous mixture as given in equation (2.6).

Finally, the temperature can be progressively estimated an iteration procedure given by

$$
T_{n+1}=T_{n}-\frac{f\left(T_{n}\right)}{f^{\prime}\left(T_{n}\right)}
$$

where $n$ is the number of iteration.

\subsection{Problem configuration initial and boundary conditions}

$\mathrm{CH}_{4}$-air diffusion flame is investigated in a one-dimensional space with $6 \mathrm{~cm}$ in length. $\mathrm{A}$ gaseous methane fuel on the left and the air on the right sides are placed in the domain. Conditions at the two ends are handled using the modified Navier-Stokes characteristic boundary conditions (NSCBC) with the nonreflecting conditions. The detailed procedure for deriving a modified NSCBC is given in (Pakdee and Mahalingam, 2003). The one-dimensional span is discretized to have 600 intervals with 601 grid points. This high resolution ensures that the reaction zone is adequately resolved. After the grid dependency has been carried out, a 600 resolution was found to be sufficient to capture the flow and thermal behavior accurately.

Regarding the mechanical condition of porous media, the resistance to the temperature cycle is the most important parameter. Therefore, ceramics that have a low thermal expansion coefficient are favorable to avoid cracks within the porous structure. The ceramic materials broadly used in applications of porous combustions are alumina $\left(\mathrm{Al}_{2} \mathrm{O}_{3}\right)$, silicon carbide $(\mathrm{SiC})$ and zirconia. In the present work, alumina and silicon carbide are investigated.

Various structures of porous media used in porous burners include packings of spheres, ceramic foams and ceramic fibres. Packings of ceramic spheres are used within the preheating region and in the heat exchanger region to enhance the heat transfer rate (Brenner et al., 2000). Within the combustion region ceramic foams or fibre structures are more efficient as their porosities are higher. This results in greater radiative heat transfer as compared with packings of spheres in addition to lower pressure loss. In the present study, the solid structure of a porous medium is assumed thermally isotropic, and the porosity considered is assumed uniform with the value of 0.8 .

\section{Results and discussion}

In order to verify the accuracy of the proposed model, the results obtained by the present study is validated against the predicted solutions for methane-air counterflow diffusion flames (Beltrame et al., 2001). In their work, a one-dimensional flame was computed using OPPDIF code (Lutz 
(a)

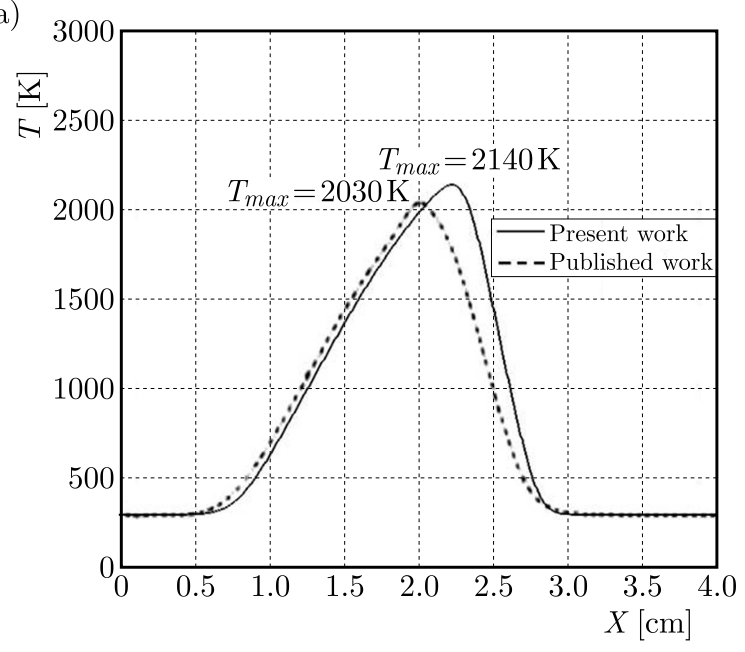

(b)

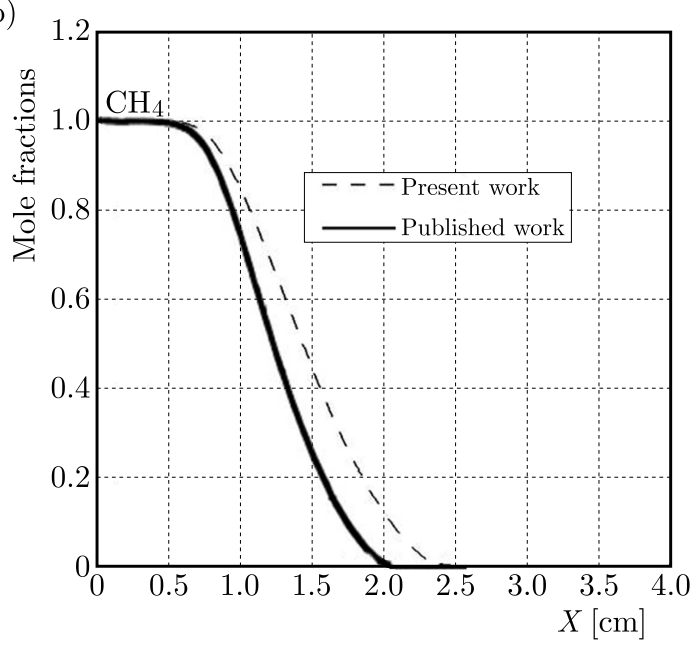

(c)

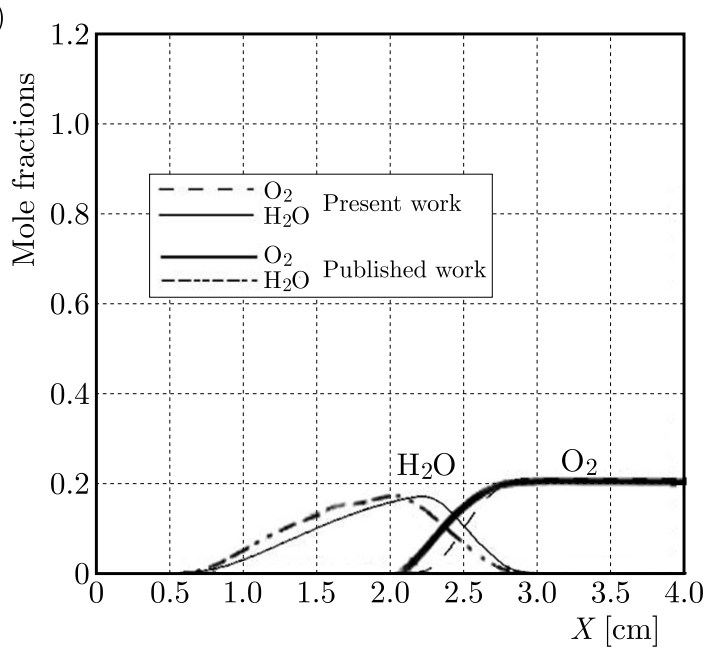

Fig. 1. Numerical solutions of the diffusion flame problem computed from the present work and the work of Beltrame et al. (2001): (a) temperature, (b) $\mathrm{CH}_{4}$ concentration, (c) $\mathrm{O}_{2}$ and $\mathrm{H}_{2} \mathrm{O}$ concentration

et al., 1996). The chemical kinetics was based on the modified GRI-MECH 2.11 (Bowman et al., 1991). The data of the stabilized flame was extracted as it propagated downstream. Comparisons of the results are shown in Figs. 1a-1c for spatial distributions of temperature and mole fractions of the reacting species. It was found that both the solutions were in good agreement with the previously published data (Beltrame et al., 2001). There appears a reaction zone in which high gradients of scalars sustain the flame stability. The predicted peak temperature, location of the flame front and species mole fractions agree reasonably well with the published data (Beltrame et al., 2001). All the profiles are slightly shifted to the right relative to the published data. Although the present calculation overpredicts the peak temperature, the difference is within $5.5 \%$. In addition to temperature, the peak values of $\mathrm{H}_{2} \mathrm{O}$ are within a $1 \%$ difference. The reasonable agreement convincingly verifies the proposed model and numerical method. Next, investigation of a porous non-premixed flame of a mixture gas is conducted with different porous materials. In what follows, the combustion process thermal and flow behavior for various cases are analyzed. The computed data are extracted and plotted. Figure 2 shows temperature distributions in three cases including the free flame and different porous ceramics which are $\mathrm{Al}_{2} \mathrm{O}_{3}$ and $\mathrm{SiC}$. The reaction zone in the porous portion appears wider than in the free space since heat is spread wider throughout the solid structure via heat conduction. As a result, fuel and air streams are continuously preheated causing the flame temperature to be higher than that of the free flame (Takeno et al., 1981). 


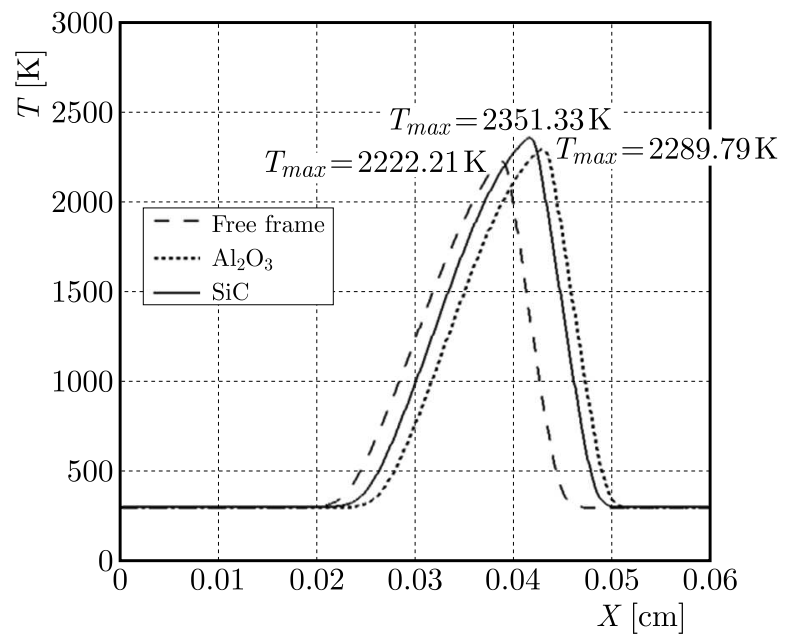

Fig. 2. Temperature profiles for different porous materials
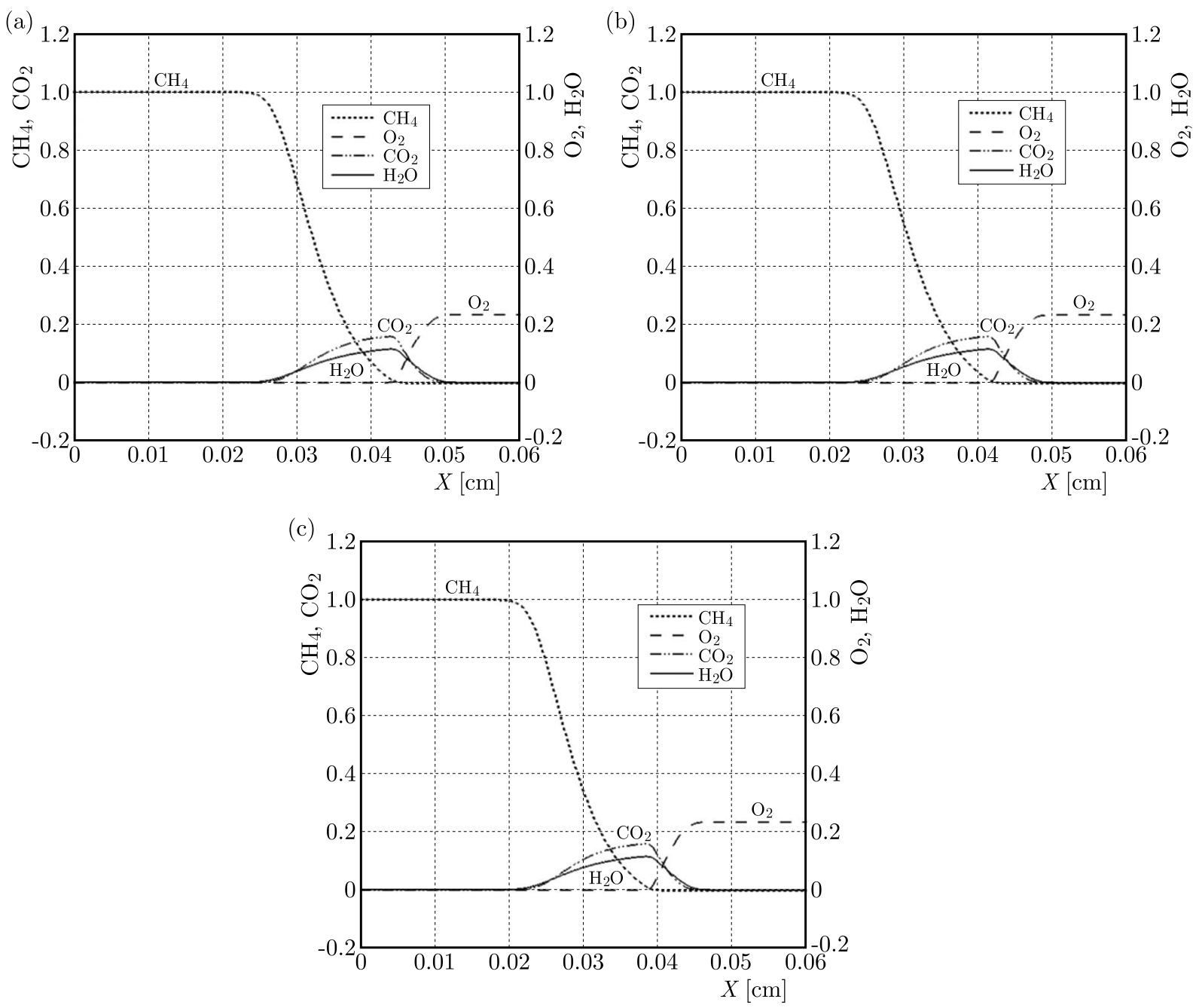

Fig. 3. Profiles of species mole fractions for porous domains: (a) $\mathrm{Al}_{2} \mathrm{O}_{3}$, (b) $\mathrm{SiC}$, (c) open space

As the materials used in the porous domain are compared, it is found that flame temperature in the $\mathrm{SiC}$ structure is greater than that occurring in the $\mathrm{Al}_{2} \mathrm{O}_{3}$ structure. This is because $\mathrm{SiC}$ has higher specific heat and lower thermal conductivity. Concentration profiles of various reacting species in terms of the mole fraction are given in Fig. 3 for both materials. It can be observed 
that the reaction zone becomes broader for the porous space than the free space. Furthermore, locations of the reaction zone found from the temperature profiles (Fig. 2) are consistent with those indicated by the concentrations profiles. In the plane of the flame front at which $\mathrm{CH}_{4}$ and $\mathrm{O}_{2}$ are completely consumed, amounts of $\mathrm{CO}_{2}$ and $\mathrm{H}_{2} \mathrm{O}$ become the highest and the temperature reaches the maximum. Additionally, it is observed that the oxidation process of the fuel is slowed down in the porous domain as compared to the free domain when the plane of the porous flame is shifted to the right relative to the plane of the free flame.

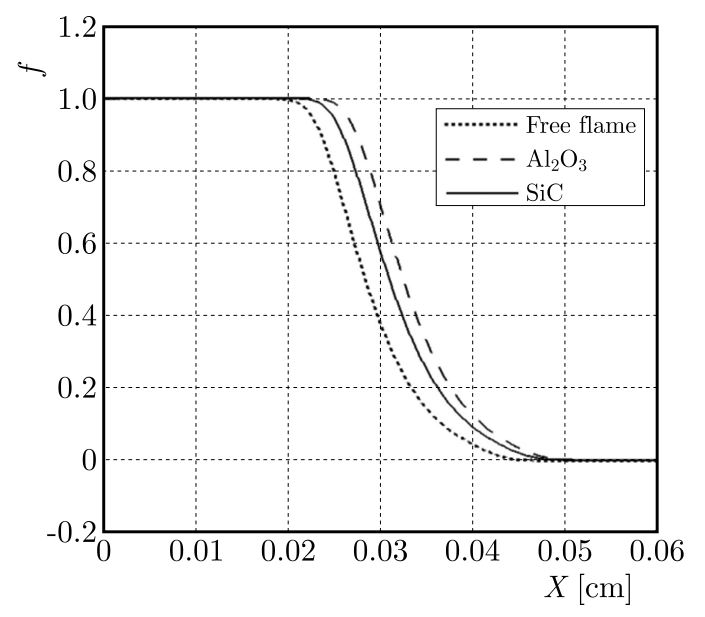

Fig. 4. Mixture fraction for various media

In regard to different materials, the reaction zone for the $\mathrm{Al}_{2} \mathrm{O}_{3}$ medium is located farther to the right. These findings are confirmed in terms of the mixture fraction in Fig. 4 as the mixture fraction of $\mathrm{Al}_{2} \mathrm{O}_{3}$ is displaced to the rightmost relative to those of $\mathrm{SiC}$ and the free flame respectively. Intense chemical reactions take place within the thin layers where the gradients of species and temperature are high, which sustains the gaseous diffusion flames. Figure 5 shows values of overall reaction rates for various cases of media. In Fig. 5 locations of the reaction rates for various cases correspond to the locations of the flame plane previously described in Fig. 3. A greater reaction rate is achieved with the presence of a porous medium due to energy recirculation by passive preheating of reactant gases. Regarding different materials, $\mathrm{CH}_{4}$ is oxidized faster in the $\mathrm{SiC}$ medium since $\mathrm{SiC}$ has thermal diffusivity higher than $\mathrm{Al}_{2} \mathrm{O}_{3}$. Moreover, the velocity field and pressure distribution throughout the domain are given in Figs. 6a and 6b, respectively. It is evident in Fig. 6a that the entire field for the $\mathrm{Al}_{2} \mathrm{O}_{3}$ case travels to the right side while it moves to the left in other cases. Furtherly, as expected, the mixture gas travels faster in the free-space domain than in the porous domain since in the porous domain there are inertial and viscous effects due to the existence of the solid structure which slows down the gas flow. These results are reasonably consistent with the location of reaction zones or flame fronts shown in the previous figures. Lastly, the gas pressure within the porous material is considerably greater than in the free space. This is mainly attributed to reduction of void volume over the entire region and the fact that the mixture gas requires greater pressure to overcome the friction force of porous surfaces.

\section{Conclusion}

A mathematical model with the proposed numerical procedure for solving an unsteady problem of nonpremixed combustion in porous media is developed. The heat transport between solid and gas phases is assumed to be local in thermal equilibrium. The accuracy of the model and the numerical method are successfully validated against the previously published data. The 
(a)

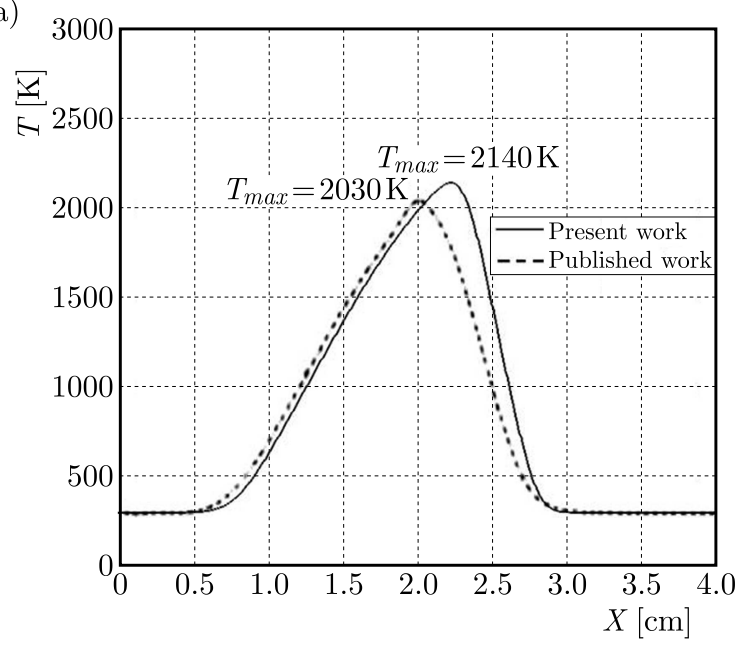

(b)

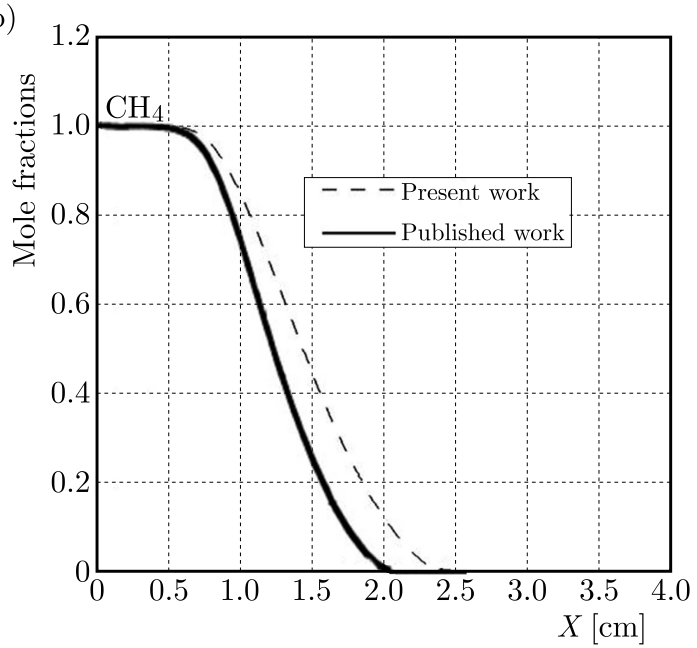

(c)

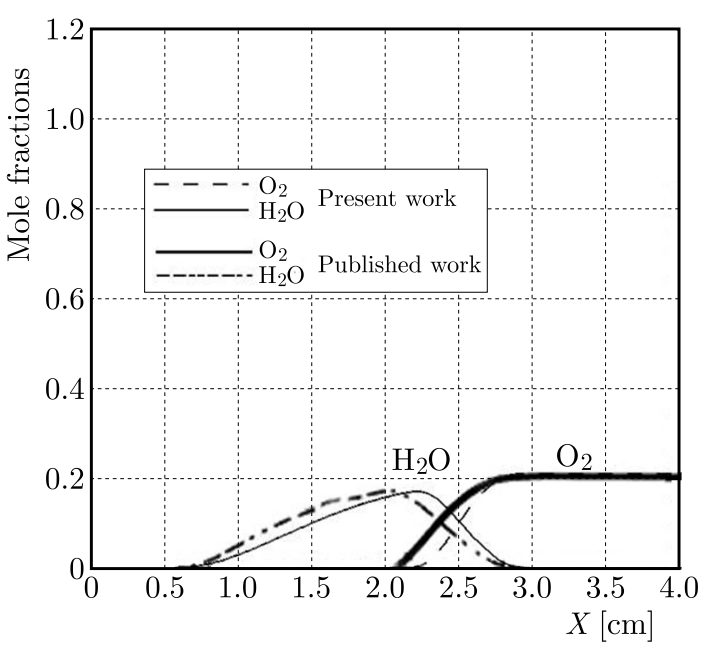

Fig. 5. Reaction rate for various media
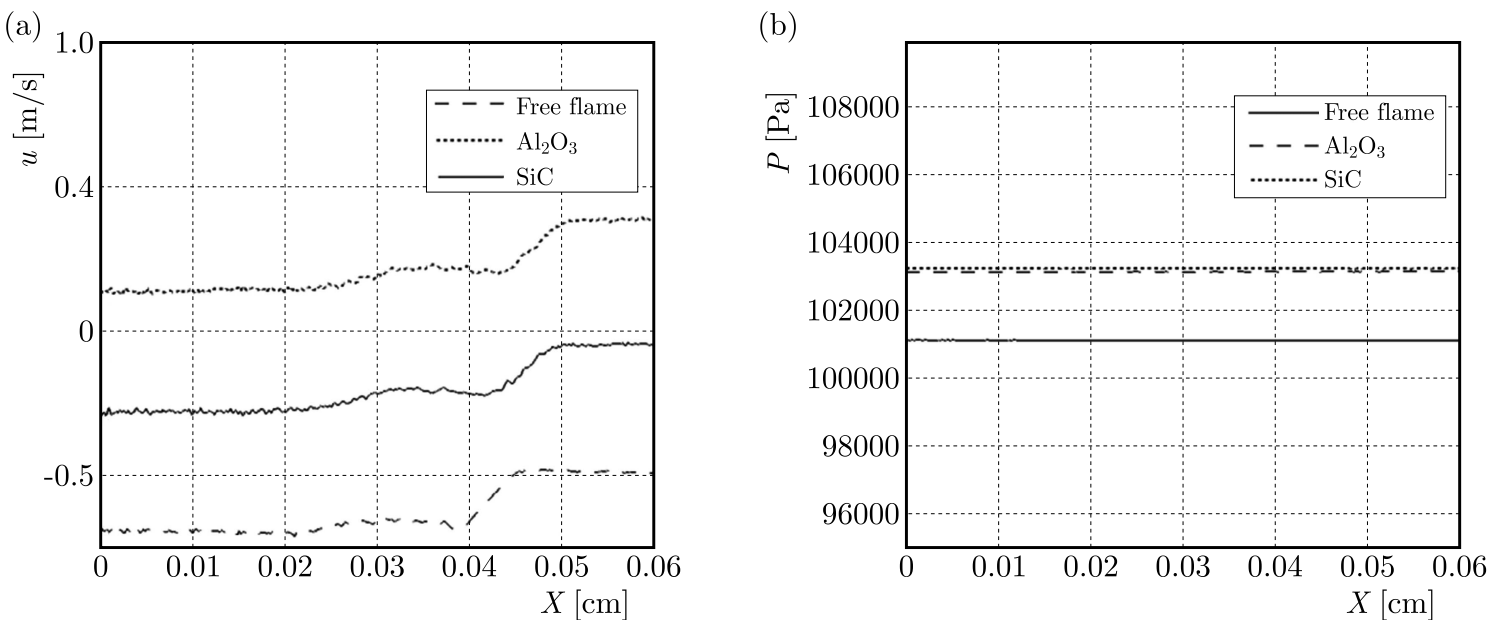

Fig. 6. Field variables: (a) velocity, (b) pressure

governing equations are discretized based on the sixth order accurate compact finite difference approximation. The third-order Runge-Kutta method is applied to advance the solutions in time. The numerical model is successfully validated by comparing the computed results with the published data (Beltrame et al., 2001). The computed data including mixture gas temperature, 
flow field and species concentrations are extracted and analyzed. The model is able to correctly describe physical behavior of premixed combustion in a porous medium. The future work is to incorporate detailed chemical kinetics for a detailed analysis of emission formation rates.

\section{References}

1. Beltrame A., Porshnev P., Merchan-Merchan W., Saveliev A., Fridman A., Kennedy L.A., Petrova O., Zhdanok S., Amouri F., Charon O., 2001, Soot and NO formation in methane-oxygen enriched diffusion flames, Combustion and Flame, 124, 295-310

2. Bowman C.T., Hanson H., Davidson D.F., Gardiner Jr. W.C., Lissiaski V., Smith G.P., Golden D.M., Frenklach M., Goldenberg M., 1991, http://www.me.Berkley.edu/gri_mech

3. Brenner G., Pickenäcker K., Pickenäcker O., Trimis D., Wawrzinek K., Webber T., 2000, Numerical and experimental investigation of matrix-stabilized methane/air combustion in porous media, Combustion and Flame, 123, 201-213

4. Bubnovich V., Henriquez L., Gnesdilov N., 2007, Numerical study of the effect of the diameter of alumina balls on flame stabilization in a porous-medium burner, Numerical Heat Transfer Part A, 52, 275-295

5. Cheatham S., Matalon M., 2000, A general asymptotic theory of diffusion flames with application to cellular instability, Journal of Fluid Mechanics, 414, 105-144

6. DE Lemos M.J.S., Silva R.A., 2006, Turbulent flow over a layer of a highly permeable medium simulated with a diffusion-jump model for the interface, International Journal of Heat and Mass Transfer, 49, 3-4, 546-556

7. Endo Kokubun M.A., Fachini F.F., Matalon M., 2017, Stabilization and extinction of diffusion flames in an inert porous medium, Proceedings of the Combustion Institute, 36, 1, 1485-1493

8. Hackert C.L., Ellzey J.L., Ezekoye O.A., 1999, Combustion and heat transfer in model two-dimensional porous burners, Combustion and Flame, 116, 177-191

9. Hosseinzadeh S., Fattahi A., Sadeghi S., Rahmani E., Bidabadi M., Zarei F., Xu F., 2020, Mathematical analysis of steady-state non-premixed multi-zone combustion of porous biomass particles under counter-flow configuration, Renewable Energy, 159, 705-725

10. Kamal M.M., Mohamad A.A., 2005, Enhanced radiation output from foam burners operating with a nonpremixed flame, Combustion and Flame, 140, 233-248

11. Kee R.J., Rupley F.M., Miller J.A., 1992, The Chemkin thermodynamic data base, Sandia National Laboratories Report, SAND-8215B

12. Klayborworn S., Pakdee W., 2019, Effects of porous insertion in a round-jet burner on flame characteristics of turbulent non-premixed syngas combustion, Case Studies in Thermal Engineering, 14, 100451

13. LELE S.K., 1992, Vortex-induced disturbance field in a compressible shear layer, Computational Physics, 103, 1, 16-42

14. Lutz A.E., Kee R.J., Grcar J.F., Rupley F.M., 1996, OPPDIF: a Fortran program for computing opposed flow diffusion flames, Sandia National Laboratories Report, SAND96-8243

15. Malico I., Zhou X.Y., Pereira J.C.F., 2000, Two-dimensional numerical study of combustion and pollutants formation in porous burners, Combustion Science and Technology, 152, 57-79

16. Marafie A., Vafai K., 2001, Analysis of non-Darcian effects on temperature differentials in porous media, International Journal of Heat and Mass Transfer, 44, 4401-4411

17. Pakdee W., Mahalingam S., 2003, Accurate method to implement boundary conditions for reacting flows based on characteristic wave analysis, Combustion Theory and Modelling, 7, 4, $705-729$ 
18. Pakdee W., Mahalingam S., 2007, Numerical investigation of turbulent non-premixed combustion of a wood pyrolysis gas, Combustion, Explosion and Shock Waves, 43, 3, 258-275

19. Pakdee W., Rattanadecho P., 2011, Natural convection in a saturated variable-porosity medium due to microwave heating, ASME Journal of Heat Transfer, 133, 062502-1-062502-8

20. Smooke M.D., Giovangigli V., 1991, Formulation of the premixed and nonpremixed test problems, [In:] Reduced Kinetic Mechanisms and Asymptotic Approximations for Methane-Air Flames, Smooke M.D. (Ed.), Lecture Notes in Physics, 384, 1-28

21. Takeno T., Sato K., Hase K., 1981, A theoretical study on an excess enthalphy flame, Proceedings of 18th International Symposium on Combustion, The Combustion Institute, Waterloo, Canada, 465-472

22. TArokh A., Mohamad A.A., Jiang L., 2009, Non-premixed $\mathrm{CH}_{4}$ combustion in a porous media, Proceedings of ASME International Mechanical Engineering Congress and Exposition, Florida, 197204

23. Trimis D., Durst F., 1996, Combustion in a porous medium - Advances and applications, Combustion Science Technology, 121, 153-168

24. Wawrzinek K., Kesting A., Künzel J., Pickenäcker K., Pickenäcker O., Trimis D., Franz M., Hartel G., 2001, Experimental and numerical study of applicability of porous combustors for HCI synthesis, Catalysis Today, 60, 393-397

25. Westbrook C.K., Dryer F.L., 1981, Simplified reaction mechanisms for the oxidation of hydrocarbon fuels in flames, Combustion Science and Technology, 27, 1, 31-43

Manuscript received April 10, 2020; accepted for print April 13, 2021 